\title{
Viewpoint
}

\section{Light Bends Itself into an Arc}

\author{
Zhigang Chen \\ Department of Physics and Astronomy, San Francisco State University, San Francisco, CA 94132, \\ USA
}

Published April 16, 2012

Mathematical solutions to Maxwell's equations suggest that it is possible for shape-preserving optical beams to bend along a circular path.

Subject Areas: Optics

\author{
A Viewpoint on: \\ Nondiffracting Accelerating Wave Packets of Maxwell's Equations \\ Ido Kaminer, Rivka Bekenstein, Jonathan Nemirovsky, and Mordechai Segev \\ Phys. Rev. Lett. 108, 163901 (2012) - Published April 16, 2012
}

equations is to assume the light waves are paraxial, meaning the angle between the wave vectors that constitute a wavepacket and the optical axis is small enough that the wave does not deviate too much from its propagation direction. Under this paraxial approximation, the resulting time-independent scalar Helmholtz equation takes the same form of the Schrödinger equation, and it was this relationship that led to the proposal that finite-power optical Airy beams could be attainable in experiments [3. (Unlike other nondiffracting beams such as the wellknown Bessel beams [7], Airy beams have a unique spatial phase structure, do not rely on simple conical superposition of plane waves, and can self-accelerate.) At small angles, Airy beams follow parabolic trajectories similar to ballistic projectiles moving under the force of gravity [8], but at large angles, beyond the paraxial approximation, they cannot maintain their shape-preserving property as they propagate. Therefore, it is important to identify mechanisms that could allow self-accelerating beams to propagate in a true diffraction-free manner even for large trajectory bending.

Several studies have searched for accelerating beams beyond the paraxial regime. In one study [9], nonparaxial Airy beams were sought as exact solutions of Maxwell's wave equation, but these beams tend to break up and decay as they propagate because parts of them consist of evanescent waves. In yet another study [10, the so-called caustic method was used to effectively stretch the paraxial Airy beams to the nonparaxial regime, but these "caustic-designed" accelerating beams don't preserve their shape like nondiffracting paraxial Airy beams. Thus, a natural question arose: Could a beam accelerate at large nonparaxial angles but still hold its shape? Since beam propagation is governed by Maxwell's equations, it was equivalent to asking: Were there any solutions to Maxwell's equations that allowed nondiffracting, self-accelerating beams?

(C) 2012 American Physical Society

DOI: 10.1103/Physics.5.44

URL: http://link.aps.org/doi/10.1103/Physics .5.44 
In their new work, Kaminer et al. report they have found shape-preserving nonparaxial accelerating beams (NABs) as a complete set of general solutions to the full Maxwell's equations. Differently from the paraxial Airy beams that accelerate along a parabolic trajectory, these nonparaxial beams accelerate in a circular trajectory.

To find the solutions for the NABs, Kaminer et al. started with the scalar Maxwell's wave equation for a given polarization, such as TE (transverse electric) polarization, where the electric field is perpendicular to the direction of the wave. Since the equation exhibits full symmetry between the $x$ and $z$ coordinates, the solutions of shape-preserving beams must have circular symmetry. The authors therefore transformed the equation into polar coordinates and looked for shape-preserving solutions where the field amplitude didn't vary with angle. In polar coordinates, the solution to the wave equation is a Bessel function; transforming back to Cartesian coordinates, the solution must be separated into forward and backward propagating waves in Fourier space. However, only the forward propagating part forms the desired accelerating beam, so the Kaminer et al. solutions are properly called "half Bessel wave packets."

The authors found a solution for TM (transverse magnetic) polarization through a similar procedure. For both TE and TM polarizations, the beams preserve their shape while the quarter-circle bending could occur after a propagation distance of just $35 \mu \mathrm{m}$. In addition, the authors studied the properties of these Bessel-like accelerating beams and found that the Poynting vector of the main lobe can turn by more than $90^{\circ} 1$.

The left part of Fig. 1 shows a typical solution of the shape-preserving beam, which can sweep out a quarter circle. It is important to note that this one-dimensional beam propagates initially along the longitudinal $z$ direction while its curved trajectory in the $x-z$ plane is determined by a Bessel function. This is quite different from the traditional nondiffracting Bessel beam, which propagates in a straight line while its two-dimensional transverse pattern follows a Bessel function [7.

As the authors point out, the nonparaxial shapepreserving accelerating beams found in their work originates from the full vector solutions of Maxwell's equation. Moreover, in their scalar form, these beams are the exact solutions for nondispersive accelerating wave packets of the most common wave equation describing timeharmonic waves. As such, this work has profound implications for other linear wave systems in nature, ranging from sound waves and surface waves in fluids to many kinds of classical waves. Furthermore, based on previous successful demonstrations of self-accelerating Airy beams [3-6], one would expect that the nonparaxial Bessel-like accelerating beams proposed in this study could be read- ily realized in experiment. Apart from many exciting opportunities for these beams in various applications, such as beams that self-bend around an obstacle (Fig. 1. right), one might expect one day light could really travel around a circle by itself, bringing the search for an

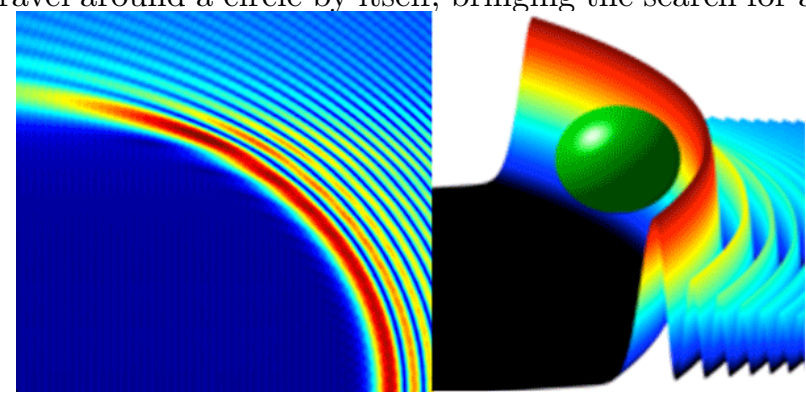

FIG. 1: Kaminer et al. showed that shape-preserving beams of light that travel along a circular trajectory emerge as solutions to Maxwell's equations. (Left) Calculated propagation of a self-bending beam. This solution assumes the wave's electric field is polarized in the transverse direction (TE polarization). (Right) Illustration of a nondiffracting beam bending around an obstacle. ((Left) Ref. 1]. (Right) Courtesy D. N. Christodoulides)

"optical boomerang" into reality.

\section{References}

[1] I. Kaminer, R. Bekenstein, J. Nemirovsky, and M. Segev, Phys. Rev. Lett. 108, 163901 (2012).

[2] M. V. Berry and N. L. Balazs, Am. J. Phys. 47, 264 (1979).

[3] G. A. Siviloglou and D. N. Christodoulides, Opt. Lett. 32, 979 (2007); G. A. Siviloglou, J. Broky, A. Dogariu, and D. N. Christodoulides, Phys. Rev. Lett. 99, 213901 (2007).

[4] J. Baumgartl, M. Mazilu, and K. Dholakia, Nature Photon. 2, 675 (2008).

[5] P. Polynkin, M. Kolesik, J. V. Moloney, G. A. Siviloglou, and D. N. Christodoulides, Science 324,229 (2009); A. Chong, W. H. Renninger, D. N. Christodoulides, and F. W. Wise, Nature Photon. 4,103 (2010).

[6] P. Zhang, S. Wang, Y. Liu, X. Yin, C. Lu, Z. Chen, and X. Zhang, Opt. Lett. 36,3191 (2011); A. Minovich, A. E. Klein, N. Janunts, T. Pertsch, D. N. Neshev, and Y. S. Kivshar, Phys. Rev. Lett. 107,116802 (2011); L. Li, T. Li, S. M. Wang, C. Zhang, and S. N. Zhu, 107,126804 (2011).

[7] J. Durnin, J. J. Miceli, Jr., and J. H. Eberly, Phys. Rev. Lett. $\mathbf{5 8 , 1 4 9 9}$ (1987).

[8] G. A. Siviloglou J. Broky, A. Dogariu, and D. N. Christodoulides, Opt. Lett. 33,207 (2008); Y. Hu, P. Zhang, C. Lou, S. Huang, J. Xu, and Z. Chen, 35,2260 (2010).

[9] A. V. Novitsky and D. V. Novitsky, Opt. Lett. 34, 3430 (2009).

[10] L. Froehly, F. Courvoisier, A. Mathis, M. Jacquot, L. Furfaro, R. Giust, P. A. Lacourt, and J. M. Dudley, Opt. Express 19,16455 (2011). 


\section{About the Author}

\section{Zhigang Chen}

Zhigang Chen received his Ph.D. in physics from Bryn Mawr College in 1995. He was
a postdoctoral research associate and then a senior research staff member at Princeton
University. He has been on the faculty in the Department of Physics and Astronomy at San
Francisco State University since 1998.

